\title{
Analytical Test on Effectiveness of MCDF Operations
}

\author{
Jun Kong ${ }^{1,2}$, Baoxue Zhang ${ }^{3}$, and Wanwu Guo ${ }^{1}$ \\ ${ }^{1}$ School of Computer and Information Science, Edith Cowan University \\ 2 Bradford Street, Mount Lawley, Western Australia 6050, Australia \\ w.guo@ecu.edu. au \\ ${ }^{2}$ Department of Computer Science, Northeast Normal University \\ 138 Renmin Street, Changchun, Jilin, China \\ kongjun@nenu.edu.cn \\ ${ }^{3}$ School of Mathematics and Statistics, Northeast Normal University \\ 138 Renmin Street, Changchun, Jilin, China \\ zhangbx@nenu.edu.cn
}

\begin{abstract}
Modified conjugate directional filtering (MCDF) is a method proposed by Guo and Watson recently for digital data and image processing. By using MCDF, directionally filtered results in conjugate directions can be not only merged into one image that shows the maximum linear features in the two conjugate directions, but also further manipulated by a number of predefined generic MCDF operations for different purposes. Although a number of cases have been used to test the usefulness of several proposed MCDF operations, and the results are 'visually' better than some conventional methods, however, no quantified analytical results on its effectiveness have been obtained. This has been the major obstacle on the decision whether it is worth developing a usable MCDF system. This paper firstly outlines a FFT-based analytical design for conducting the tests, and then presents the results of applying this analytical design to the analysis of $\mathrm{MCDF}(\operatorname{add} 1)$ operation for an image of digital terrain model in central Australia. The test verifies that the MCDF(add1) operation indeed overcomes the two weaknesses of using the conventional directional filtering in image processing, i.e., separation in presentation of processed results in different directions, and significant loss in low-frequency components. Therefore, the MCDF method is worth for further development.
\end{abstract}

\section{Introduction}

Guo and Watson [1] recently reported the trial work on using a method called the modified conjugate directional filtering (MCDF) for digital image processing. By using MCDF, directionally filtered results in conjugate directions can be not only merged into one image that shows the maximum linear features in the two conjugate directions, but also further manipulated by a number of predefined MCDF operations for different purposes. MCDF is modified from the previous proposal named conjugate directional filtering (CDF) [2], because further study reveals that the CDF has two weaknesses, i.e., a weighting system for further data manipulation during the operation was not considered; CDF-processed images often lack contrast depth because most background information is removed as a result of applying directional filtering. 
MCDF overcomes these problems by superimposing the weighted CDF data onto the original data. In this way, not only further enhanced by a weighting factor are the conjugated features, but also retained is all the information on the original image. By introducing these two considerations into the CDF, MCDF becomes much more powerful [1][3].

Although a number of cases have been used to test the usefulness of several proposed MCDF operations, and the results are 'visually' better than some conventional methods [4][5], however, no quantified analytical results on its effectiveness have been obtained. This has been the major obstacle on the decision whether it is worth the development of a usable MCDF system. Our recent study on analytical test design and experiments on MCDF operations have led to the acquisition of some positive and encouraging results. In this paper, we first briefly present the concepts of the MCDF operations, and then outline the analytical design for the tests. Due to the restriction on the paper length, we only apply this analytical design to the analysis of MCDF(add1) operation in this paper. An image of digital terrain model in central Australia is used to analyze the effectiveness of the MCDF(add1) operation.

\section{MCDF Operations and Analytical Test Design}

Directional filtering is used to enhance linear features in a specific direction [4][5][6]. In some cases, identifying conjugate linear information on an image is particularly concerned. Directional filtering can be made in two specific conjugate directions to enhance these conjugate features. Normally the filtered results from the two conjugate directions are shown on two separate images. This is inconvenient for revealing the relationships between linear features in the two conjugate directions. The linear enhancement using directional filtering is achieved by constraining or removing the textural features or low-frequency components from the original image to outline the structural features or high-frequency components contained in the original image. Thus, directionally filtered image often lacks contrast depth because most background information is removed.

These two weaknesses of using the conventional directional filtering are overcome by MDCF method, which firstly combines two (or more) directional-filtered results in conjugate directions into one image that exhibits the maximum linear features in the two conjugate directions, and secondly retains the background information by superimposing the directionally filtered data onto the original data. Therefore, the analytical tests should be designed in a way through which these two improvements can be clearly revealed.

Assuming $f_{0}$ to be the original data file, $f_{1}$ and $f_{2}$ to be the directional-filtered data files in the two conjugate directions, the general operation of the MCDF can be expressed as [1]

$$
\mathrm{MCDF}=W_{0} \cdot f_{0}+F_{2}\left[W_{1} \cdot F_{l}\left(f_{l}\right), W_{2} \cdot F_{l}\left(f_{2}\right)\right] ;
$$

where $W_{0}, W_{1}$ and $W_{2}$ are selective constants; $F_{0}, F_{1}$ and $F_{2}$ are pre-defined generic functions. Consequently, some MCDF operations are defined using formula (1) as

$$
\operatorname{MCDF}(\operatorname{add} 1)=W_{0} f_{0}+W_{1} ; f_{1}+W_{2} f_{2}
$$




$$
\begin{gathered}
\operatorname{MCDF}(\operatorname{add} 2)=W_{0}^{\prime} f_{0}+\operatorname{abs}\left(W_{1}^{\prime} f_{1}+W_{2}^{\prime} f_{2}\right) ; \\
\operatorname{MCDF}(\operatorname{add} 3)=W_{0}^{\prime} f_{0}+W_{1} \cdot \operatorname{abs}\left(f_{1}\right)+W_{2}^{\prime} \operatorname{abs}\left(f_{2}\right) ; \\
\operatorname{MCDF}(\max 1)=F_{0}\left(W_{0}^{\prime} f_{0}\right)+\max \left(W_{1}^{\prime} f_{1}, W_{2}^{\prime} f_{2}\right) ; \\
\operatorname{MCDF}(\max 2)=F_{0}\left(W_{0}^{\prime} f_{0}\right)+\max \left[W_{1}^{\prime} \operatorname{abs}\left(f_{1}\right), W_{2}^{\prime} \operatorname{abs}\left(f_{2}\right)\right] ; \\
\operatorname{MCDF}(\operatorname{ampl})=W_{0}^{\prime} f_{0}+\operatorname{sqrt}\left(W_{1}^{\prime} f_{1}^{\prime} f_{1}+W_{2}^{\prime} f_{2}^{\prime} f_{2}\right) .
\end{gathered}
$$

We propose a design for taking the analytical tests shown in Figure 1. Firstly, original image and each of its MCDF-processed images are input individually to a processing unit for analysis using fast Fourier transform (FFT). The output from this FFT analysis includes the 2D Cartesian spectrum, and radial spectrum of the corresponding input image [7]. Compared with the outcomes of the original and MCDF(add1) images, the 2D Cartesian spectrum is used to directly identify whether the MCDF operations indeed have brought enhanced information in the conjugate directions into MCDF-processed images; the radial spectrum is used to quantify whether the MCDF-processed images have retained the background information or low-frequency components of the original image while the structural features or highfrequency components are enhanced.

To make the analytical results acceptable as widely as possible, the FFT analysis of our tests are carried out using FFT functions provided by Matlab [8][9]. Next section reports the test results of $\mathrm{MCDF}(\operatorname{add} 1)$ operation using this test design on an image of digital terrain model.

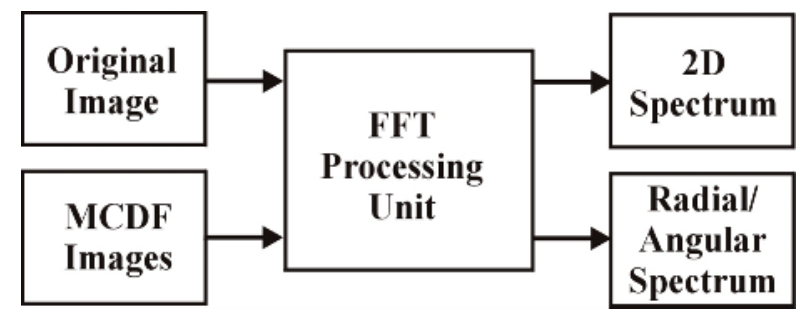

Fig. 1. Schematic diagrams of the design for analytical test of MCDF operations

\section{Test Results of Image of Digital Terrain Model (DTM)}

Figure $2 \mathrm{a}$ is the original image of digital terrain model (DTM) of a desert in central Australia. This region has a relatively low topographic relief $(<200 \mathrm{~m})$. The dark colors indicate the desert whereas the light colors indicate the highlands or hills in the desert. NW-trending features are prominent whereas some subtle NE-trending structures also exist. However, with the dominance of dark colors in the desert, detailed features in the desert are hardly seen on the original image. Figure $2 b$ shows the $2 \mathrm{D}$ 
Cartesian spectrum of this image. The conjugated NW-and-NE trending features are reflected as alignments mixed within the elongated frequency zone in Figure 2b. Figure $2 \mathrm{c}$ shows that the intensity of different frequency components decreases dramatically with the increase in frequency, with the intensity of high-frequency components ( $>400 \mathrm{Rad} / \mathrm{s}$ ) being less than $1 \%$ of the maximum intensity.

Figure 3a shows the image processed with $\mathrm{MCDF}(\operatorname{add} 1)$. NE and NW directions are selected as the two conjugate directions for directional filtering. The combination of $W_{0}=1, W_{N W}=W_{N E}=2, F_{1}=F_{2}=1$ generates an image on which many NE and NW linear features are outlined in the 'dark' desert. The conjugated NW-and-NE trending features are clearly reflected as alignments which separate the original elongated frequency zone into two fan-shaped sub-zones in Figure $2 \mathrm{~b}$. This indicates that this MCDF operation indeed enhanced the features in these conjugate directions. The total area of these 2 sub-zones is larger than that of the elongated zone in the Figure $1 \mathrm{~b}$, which implies the enhancement of high-frequency components contained in the original image. Figure $3 \mathrm{c}$ further shows that the high-frequency components have been intensified to $12 \%$ of the maximum intensity. The intensity of medium-frequency components is also increased whereas the expected low-frequency components are retained with the same intensity.

\section{Discussions and Conclusion}

To verify that the MCDF(add1) operation indeed enhances the conjugated features in both NE and NW directions in the image, comparison can be made between the two spectra of the original and MCDF(add1) images (Fig. 2b \& Fig. 3b). In the spectrum of the original image, NE and NW trending information is mixed with other components in an elongated frequency zone, distinguished by locally 'light-colored' alignments in these two conjugated directions from other components. However, in the spectrum of the MCDF(add1) image, NE and NW trending information is distinguished by clear 'light-dark' margins along these two conjugated directions, which indicates the significant separation between the enhanced high-frequency NE and NW trending features from their surrounding low-frequency components. As expected, the MCDF(add1) image (Fig. 3a) indeed has shown more NE and NW trending information than that in the original image (Fig. 2a).

To verify that the MCDF(add1) operation not only enhances the conjugated features in both NE and NW directions in the image, but also retains the low-frequency information in the original image, we use the statistical results of radial spectra of both the original and MCDF(add1) images (Table 1) to outline the facts. It is evident that the MCDF(add1) operation has enhanced the highest-frequency component by 9 times from its relative intensity of $0.5 \%$ in the original image to $4.5 \%$ in the MCDF(add1) image. This is achieved by keeping almost no change in the maximum intensity and standard deviation in both images, which means that there is almost no loss in low-frequency components in the MCDF(add1) image. The medium-frequency components are also intensified from $6.3 \%$ in the original image to $16.9 \%$ in the MCDF(add1) image, an increase of 2.7 times. By keeping the same low-frequency components, bringing a moderate increase in medium-frequency components, and elevating high-frequency components by at least 9 times, all together the $\mathrm{MCDF}(\mathrm{add} 1)$ operation makes not only features in the NE and NW directions in the 
MCDF(add1) image look more prominent, but also the whole image appear richer in contrast depth and thus more smooth.

Table 1. Statistics of radial spectra of the original and MCDF(add1) images

\begin{tabular}{|c|c|c|c|c|}
\hline \multirow{2}{*}{ Statistics } & \multicolumn{2}{|c|}{ Original image } & \multicolumn{2}{c|}{ MCDF(add) image } \\
\cline { 2 - 5 } & Absolute & $\begin{array}{c}\text { Relative } \\
\text { (x/Max) }\end{array}$ & $\begin{array}{c}\text { Absolute } \\
\text { intensity }\end{array}$ & $\begin{array}{c}\text { Relative } \\
\text { (x/Range) }\end{array}$ \\
\hline $\begin{array}{c}\text { Min (high-frequency } \\
\text { components) }\end{array}$ & 826 & $0.5 \%$ & 7446 & $4.5 \%$ \\
\hline $\begin{array}{c}\text { Max (low-frequency } \\
\text { components) }\end{array}$ & 164359 & $100 \%$ & 164345 & $100 \%$ \\
\hline $\begin{array}{c}\text { Median (medium- } \\
\text { frequency components) }\end{array}$ & 10372 & $6.3 \%$ & 27810 & $16.9 \%$ \\
\hline $\begin{array}{c}\text { Mean (average fre- } \\
\text { quency components) }\end{array}$ & 22267 & $13.5 \%$ & 34883 & $21.2 \%$ \\
\hline Std & 26299 & $16 \%$ & 25492 & $15.5 \%$ \\
\hline
\end{tabular}

Our FFT analysis on the DTM image proves that the MCDF(add1) operation indeed overcomes the two weaknesses of using the conventional directional filtering in image processing, i.e., separation in presentation of processed results in different directions, and significant loss in low-frequency components. Although the results of using MCDF(add1) are presented here only, tests over other MCDF operations also reveal the similar results (Table 2). Therefore, the MCDF method is worth for further development.

Table 2. Statistics of radial spectra of an aerial photograph and its MCDF(add2) image

\begin{tabular}{|c|c|c|c|c|}
\hline \multirow{2}{*}{ Statistics } & \multicolumn{2}{|c|}{ Original image } & \multicolumn{2}{c|}{ MCDF(add) image } \\
\cline { 2 - 5 } & Absolute & $\begin{array}{c}\text { Relative } \\
\text { (x/Max) }\end{array}$ & $\begin{array}{c}\text { Absolute } \\
\text { intensity }\end{array}$ & $\begin{array}{c}\text { Relative } \\
\text { (x/Range) }\end{array}$ \\
\hline $\begin{array}{c}\text { Min (high-frequency } \\
\text { components) }\end{array}$ & 12297 & $7.8 \%$ & 32974 & $20.9 \%$ \\
\hline $\begin{array}{c}\text { Max (low-frequency } \\
\text { components) }\end{array}$ & 158111 & $100 \%$ & 158105 & $100 \%$ \\
\hline $\begin{array}{c}\text { Median (medium- } \\
\text { frequency components) }\end{array}$ & 36711 & $23.2 \%$ & 53042 & $33.5 \%$ \\
\hline $\begin{array}{c}\text { Mean (average fre- } \\
\text { quency components) }\end{array}$ & 43556 & $27.5 \%$ & 57933 & $36.6 \%$ \\
\hline Std & 28270 & $17.9 \%$ & 22712 & $14.4 \%$ \\
\hline
\end{tabular}




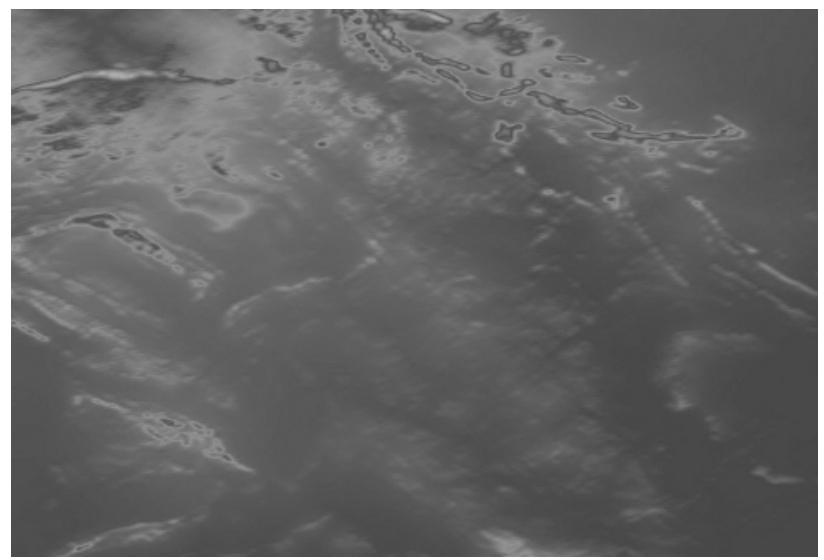

a

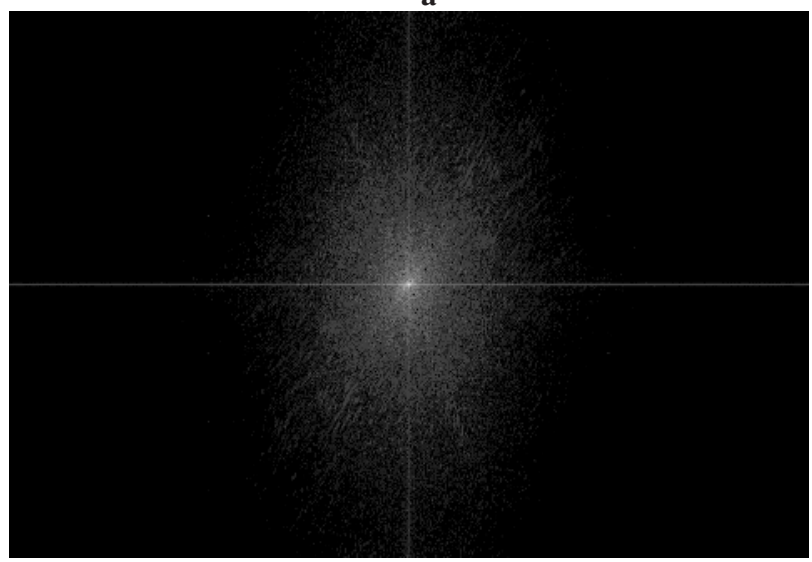

b

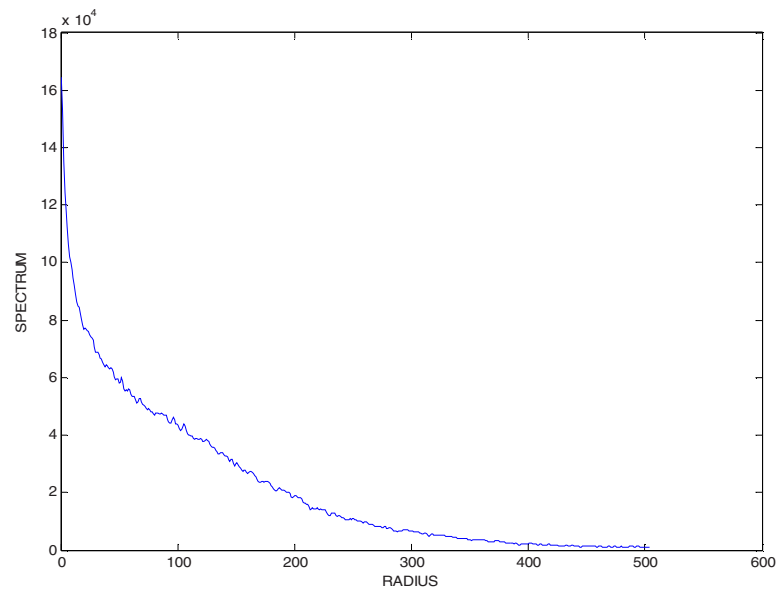

c

Fig. 2. Original image (a), 2D spectrum (b), and radial spectrum (c) 


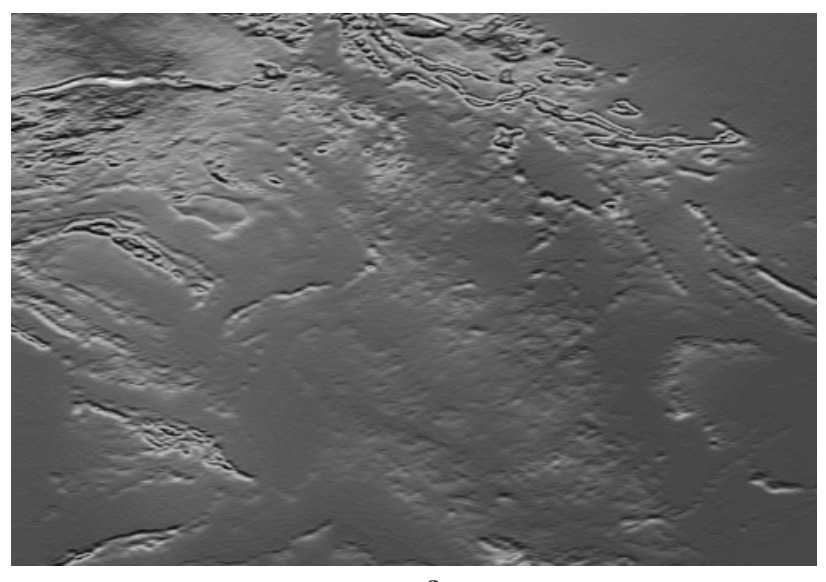

a

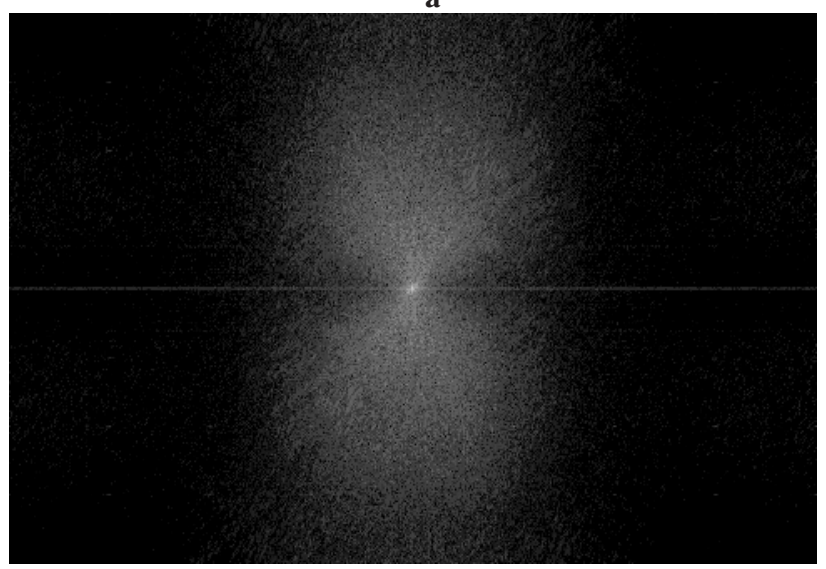

b

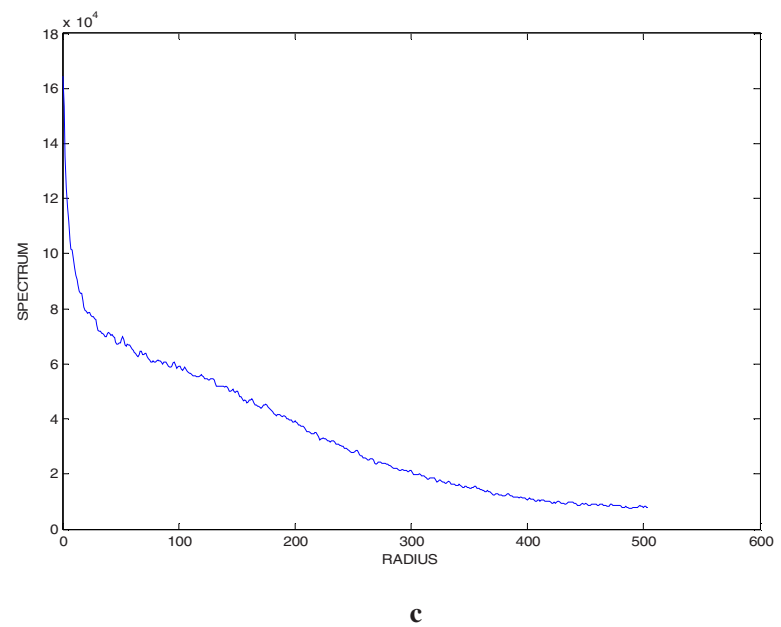

Fig. 3. MCDF(add1) image (a), 2D spectrum (b), and radial spectrum (c) 
Acknowledgement. We are grateful to the Northern Territory Geological Survey of Department of Mines and Energy of Australia for providing us with the DTM data. The Faculty of Communication, Health and Science of the Edith Cowan University is thanked for supporting this research project. The constructive comments made by the anonymous referees are acknowledged.

\section{References}

1. Guo, W., Watson, A.: Modification of Conjugate Directional Filtering: from CDF to MCDF. Proceedings of IASTED Conference on Signal Processing, Pattern Recognition, and Applications. Crete, Greece (2002) 331-334.

2. Guo, W., Watson, A.: Conjugated Linear Feature Enhancement by Conjugate Directional Filtering. Proceedings of IASTED Conference on Visualization, Imaging and Image Processing. Marbella, Spain (2001) 583-586.

3. Watson. A., Guo, W.: Application of Modified Conjugated Directional Filtering in Image Processing. Proceedings of IASTED Conference on Signal Processing, Pattern Recognition, and Applications. Crete, Greece (2002) 335-338.

4. Jahne, B.: Digital Image Processing: Concepts, Algorithms and Scientific Applications. Springer-Verlag, Berlin Heidelberg (1997).

5. Proakis, J.G., Manolakis, D.G.: Digital Signal Processing: Principles, Algorithms and Applications. Prentice-Hall, Upper Saddle River New York (1996).

6. Richards, J.A.: Remote Sensing Digital Image Analysis. Springer-Verlag, Berlin Heidelberg (1993).

7. Gonzalez, R.C., Woods, R.E.: Digital Image Processing, Prentice Hall (2002).

8. Hanselman, D., Littlefield, B.R.: Mastering MATLAB 6. Prentice Hall (2001).

9. Phillips, C.L., Parr, J.M., Riskin, E.A.: Signals, Systems, and Transforms. Prentice Hall (2003). 